\title{
Efficient voice activity detection algorithm using long-term spectral flatness measure
}

\author{
Yanna Ma* and Akinori Nishihara
}

\begin{abstract}
This paper proposes a novel and robust voice activity detection (VAD) algorithm utilizing long-term spectral flatness measure (LSFM) which is capable of working at $10 \mathrm{~dB}$ and lower signal-to-noise ratios(SNRs). This new LSFM-based VAD improves speech detection robustness in various noisy environments by employing a low-variance spectrum estimate and an adaptive threshold. The discriminative power of the new LSFM feature is shown by conducting an analysis of the speech/non-speech LSFM distributions. The proposed algorithm was evaluated under 12 types of noises (11 from NOISEX-92 and speech-shaped noise) and five types of SNR in core TIMIT test corpus. Comparisons with three modern standardized algorithms (ETSI adaptive multi-rate (AMR) options AMR1 and AMR2 and ITU-T G.729) demonstrate that our proposed LSFM-based VAD scheme achieved the best average accuracy rate. A long-term signal variability (LTSV)-based VAD scheme is also compared with our proposed method. The results show that our proposed algorithm outperforms the LTSV-based VAD scheme for most of the noises considered including difficult noises like machine gun noise and speech babble noise.
\end{abstract}

\section{Introduction}

Voice activity detection (VAD) is a method to discriminate speech segments from input noisy speech. It is an integral part to many speech and audio processing applications and is widely used within the field of speech communication for achieving high coding efficiency and low bit rate transmission. Examples include noise reduction for digital hearing aid devices [1], mobile communication services [2], voice recognition systems [3], compression [4], and speech coding [5].

A typical VAD system consists of two core parts: feature extraction and speech/non-speech decision mechanism. Researchers have proposed a variety of features exploiting different properties of speech and noise to achieve better VAD performance. In early VAD algorithms, shortterm energy [6] and zero-crossing rate [7] were widely used features because of their simplicity. However, the performance degrades easily when faced with low signalto-noise ratio (SNR) or non-stationary background noise. To solve this problem, robust acoustic features such as spectrum [8], autocorrelation [9], power in the bandlimited region [10], and higher-order statistics [11] have

*Correspondence: mayanna@nh.cradle.titech.ac.jp

Department of Communications and Integrated Systems, Tokyo Institute of Technology, Tokyo 152-8552, Japan been proposed for VAD. Most of those methods assume the background noise to be stationary during a certain period; thus, they are sensitive to changes in SNR of the observed signal. Some works $[12,13]$ proposed noise estimation and adaptation for improving VAD robustness, but those methods are computationally expensive. Most of those features mentioned work sufficiently well in stationary noise and higher than 10-dB SNR cases. When facing with lower SNR cases or when the background noise contains complex audible events appearing occasionally, such as babble noise in a cafeteria and machinery noise in a factory, there will be cases when most of the speech spectrum is corrupted, which destroys the overall statistical as well as structural properties of the speech signal [14]. In general, VAD algorithms based on a particular feature or specific set of features are still far from efficient especially when they are operating in adverse acoustic conditions. Therefore, the VAD algorithm in low SNRs and some specific noises such as speech babble noise and machine gun noise still remains challenging and requires the design of further robust features and algorithms.

All VAD features mentioned are extracted from the short-term analysis frames (usually 20 to $40 \mathrm{~ms}$ ), and decisions are made at each frame. In contrast with the use of frame level features, Ramirez et al. [12] proposed

\section{Springer}

(c) 2013 Ma and Nishihara; licensee Springer. This is an Open Access article distributed under the terms of the Creative Commons Attribution License (http://creativecommons.org/licenses/by/2.0), which permits unrestricted use, distribution, and reproduction in any medium, provided the original work is properly cited. 
the use of a long-term spectral divergence feature to discriminate speech from noise. It requires average noise spectrum magnitude information which is not accurately available in practice. Moreover, Ghosh et al. [15] proposed a long-term signal variability (LTSV)-based VAD which uses a very long window to estimate the averaged spectrogram as well as for computing long-term entropies of each frequency band. This LTSV-based VAD yields a great improvement for SNRs smaller than $5 \mathrm{~dB}$ but becomes saturated when SNRs are higher than $5 \mathrm{~dB}$.

Spectral flatness is a measure of the width, uniformity, and noisiness of the power spectrum. A high spectral flatness indicates that the spectrum has a similar amount of power in all spectral bands, and the graph of the spectrum would appear relatively flat and smooth; this would sound similar to white noise. A low spectral flatness indicates that the spectral power is less uniform in frequency structure, and this would typically sound like speech. Therefore, the analysis over a long window for exploiting the spectral flatness of the signal will be beneficial for distinguishing speech from noise. In this paper, we propose a novel VAD algorithm based on long- term spectral flatness measure (LSFM). The discriminative power of the proposed LSFM feature will be verified by researching the distribution of LSFM measure for speech and non-speech in terms of their misclassification rate for various noises. We have experimentally evaluated its performance under a variety of noise types and SNR conditions.

The structure of the rest of this article is arranged as follows. Section 2 discusses the LSFM feature and its discriminative ability. Section 3 presents the proposed LSFM-based VAD algorithm including the choice for proper parameters and the design of an adaptive threshold. Section 4 contains the speech and noise database and metrics used in the evaluation. Section 5 provides the experimental results. Finally, a conclusion of this work and the discussion are given in Section 6.

\section{Long-term spectral flatness measure and its discriminative power}

Speech is a highly non-stationary signal, while background noise can be considered to be stationary over relatively long periods. The rationale behind the LSFM

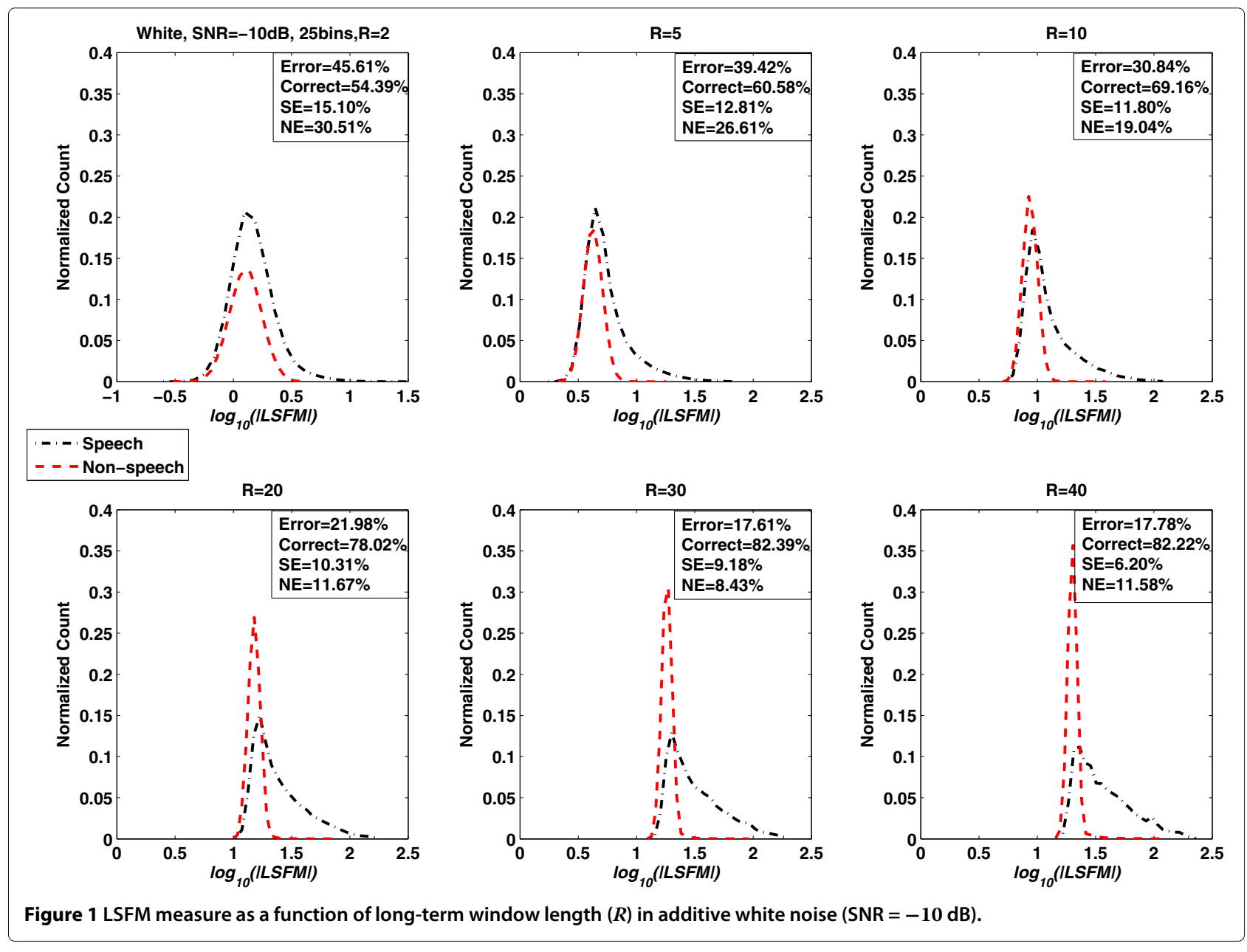


feature is that the observed signal spectrum evinces more structure when the signal of interests is present compared to when it is absent. This increase in the structure of the signal may be characterized by a reduction in the flatness of the magnitude spectrum of the short-time Fourier representation of the signal [16].

\subsection{Long-term spectral flatness measure}

The LSFM feature is computed using the spectra of the last $R$ frames of the input signal $x(n)$. The LSFM feature, $L_{x}(m)$, at the $m$ th frame and across all the chosen frequency is then calculated by dividing the geometric mean of the power spectrum by the arithmetic mean of the power spectrum. To expand the dynamic range, it is measured on a logarithmic scale, ranging from zero to minus infinity as:

$$
L_{x}(m)=\sum_{k} \log _{10} \frac{\operatorname{GM}\left(m, \omega_{k}\right)}{\operatorname{AM}\left(m, \omega_{k}\right)}
$$

where the geometric mean $\operatorname{GM}\left(m, \omega_{k}\right)$ and arithmetic mean $\operatorname{AM}\left(m, \omega_{k}\right)$ of the power spectrum is calculated as:

$$
\begin{aligned}
& \operatorname{GM}\left(m, \omega_{k}\right)=\sqrt[R]{\prod_{n=m-R+1}^{m} S\left(n, \omega_{k}\right),} \\
& \operatorname{AM}\left(m, \omega_{k}\right)=\frac{1}{R} \sum_{n=m-R+1}^{m} S\left(n, \omega_{k}\right) .
\end{aligned}
$$

The short-time spectrum $S\left(n, \omega_{k}\right)$ used in this research is estimated using the Welch-Bartlett method which averages the spectral estimates of $M$ consecutive frames. The expressions are

$$
\begin{aligned}
& S\left(n, \omega_{k}\right)=\frac{1}{M} \sum_{p=n-M+1}^{n}\left|X\left(p, \omega_{k}\right)\right|^{2}, \\
& X\left(p, \omega_{k}\right)=\sum_{l=(p-1) N_{\mathrm{sh}}+1}^{N_{w}+(p-1) N_{\mathrm{sh}}} w\left(l-(p-1) N_{\mathrm{sh}}-1\right) x(l) e^{-j \omega_{k} l},
\end{aligned}
$$

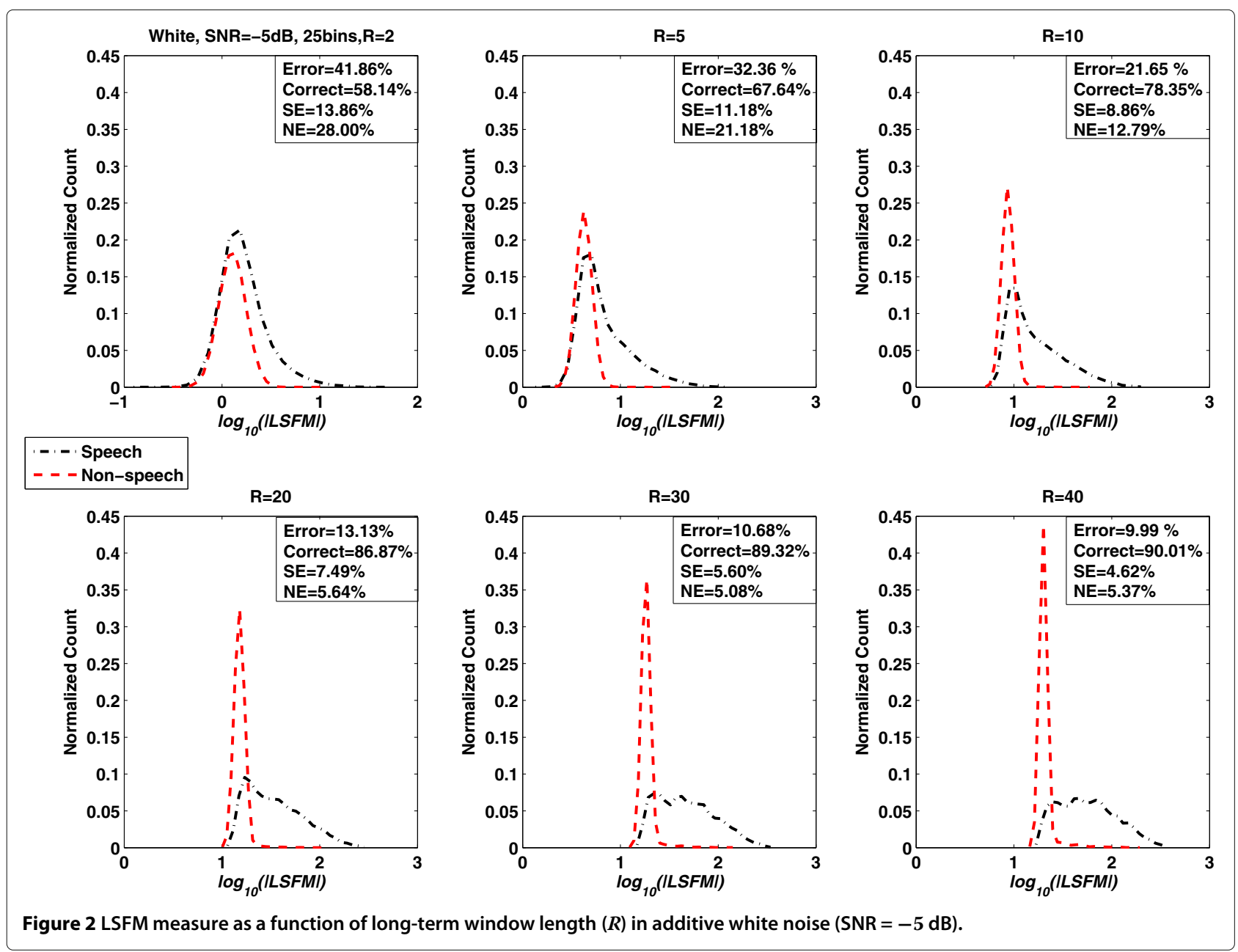


where $X\left(p, \omega_{k}\right)$ is the short-time Fourier transform coefficient at frequency $\omega_{k}$ of the $p$ th frame. $w(i)$ is the short-time Hann window, where $i \in\left[0, N_{w}\right) . N_{w}$ is the frame length and $N_{\mathrm{sh}}$ is the frame shift duration in terms of samples.

According to AM-GM inequality, the geometric mean, $\operatorname{GM}\left(m, \omega_{k}\right)$, is smaller than or equal to the arithmetic mean, $\operatorname{AM}\left(m, \omega_{k}\right)$, with equality being achieved if and only if all $S\left(n, \omega_{k}\right)$ are the same. Therefore, from Equation 1, we can conclude that the LSFM feature, $L_{x}(m)$, is in the range $(-\infty, 0]$ with the maximum value acquired when the geometric mean is equal to the arithmetic mean.

\subsection{The LSFM feature distributions of speech and non-speech}

In this subsection, the distributions of the LSFM feature are investigated in order to clarify the motivation for utilizing the proposed LSFM feature as a VAD algorithm and demonstrate the discriminative power of this feature.

The test set consisting of 16 individual speakers (8 male, 8 female), each speaking 10 phonetically balanced English sentences, is randomly chosen from the TIMIT training corpus [17]. The LSFM feature values were computed at every frame from noisy speech. The LSFM measure, $L_{x}(m)$, is considered to be $L_{S+N}(m)$ if there are speech samples between $(m-R+1)$ th and $m$ th frame. Otherwise, it is decided to be $L_{N}(m)$. The overlap area of the two distributions $\left(L_{S+N}\right.$ and $\left.L_{N}\right)$ is considered to be the error caused by misclassification. The lower the misclassification rate is, the better the separation. The sampling frequency of the test signal is $16 \mathrm{kHz}$, and the Hann window has a length of $20 \mathrm{~ms}$ and a $10-\mathrm{ms}$ shift. $M$ is fixed to be 10 , and $\left\{\omega_{k}\right\}$ is uniformly distributed between the frequency range $500 \mathrm{~Hz}$ to $4 \mathrm{kHz}$. The total misclassification error among these realizations of $L_{S+N}$ and $L_{N}$ was computed by comparing with the phonetic level transcription [17] of the TIMIT training corpus.

First, the distributions of the LSFM feature as a function of the long-term window length $(R=2,5,10,20$, 30 , and 40$)$ for white noise at five SNR levels $(-10,-5$, 0,5 , and $10 \mathrm{~dB}$ ) were studied. The results are shown in Figures 1, 2, 3, 4, and 5. The total misclassification

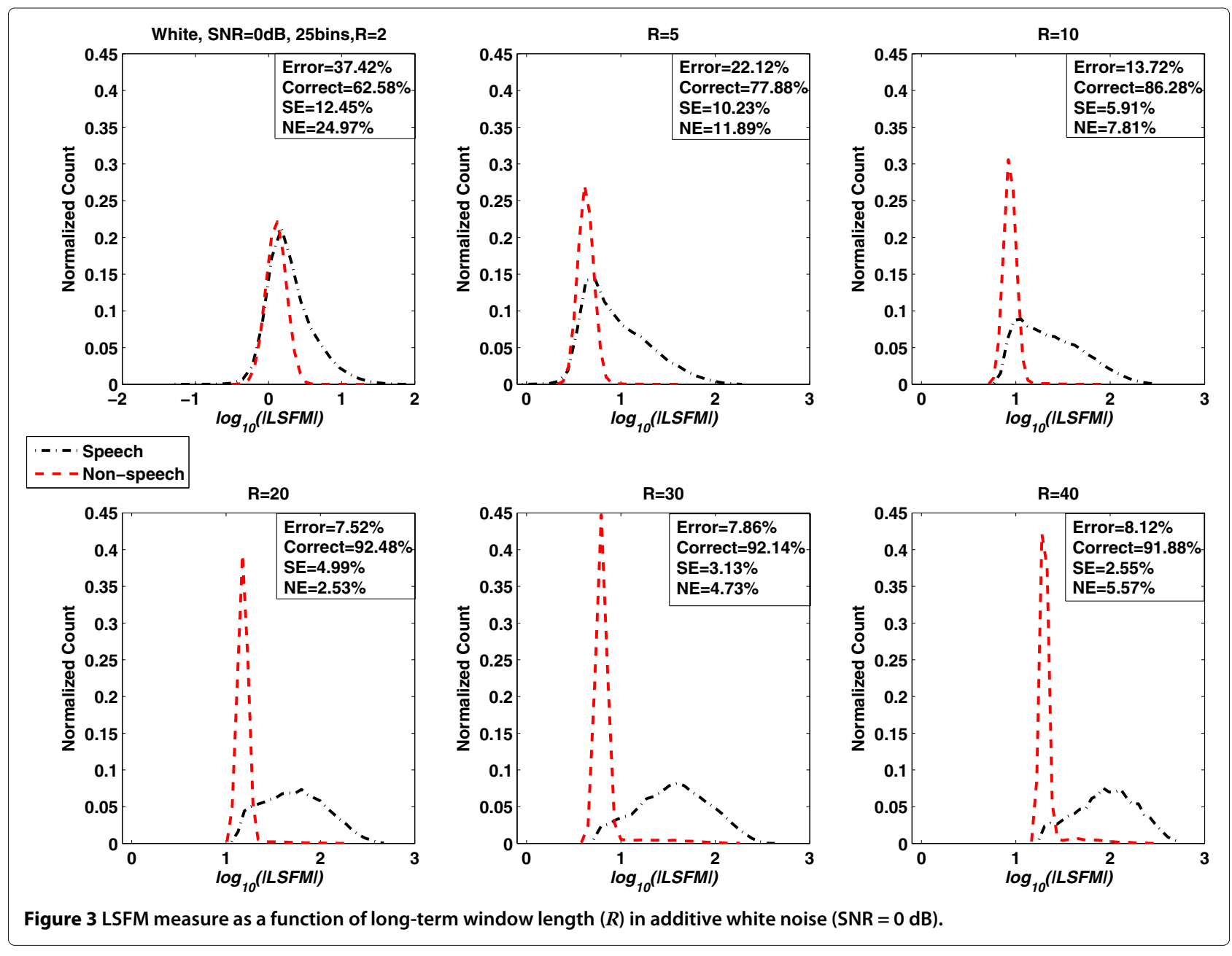




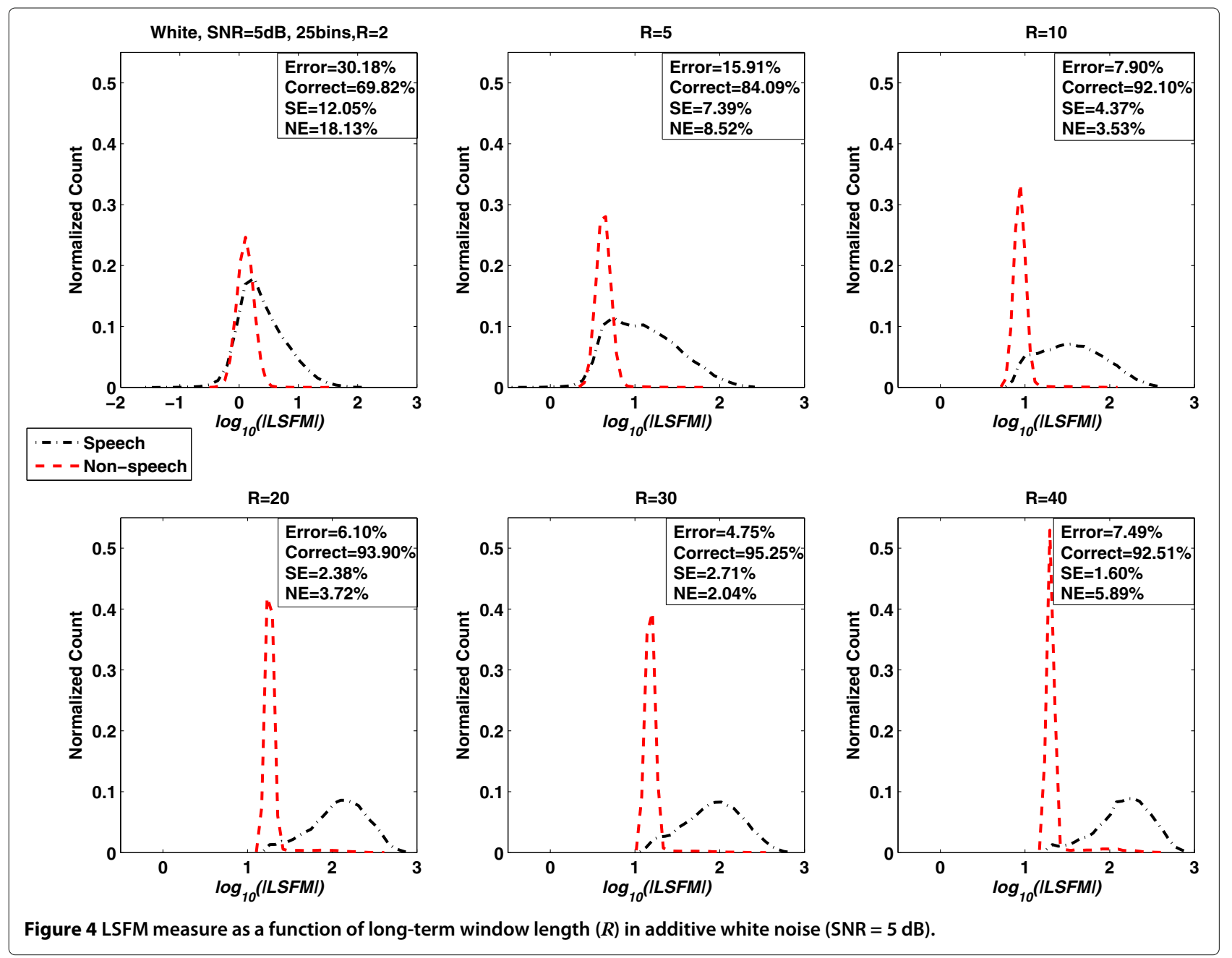

error (Error), accuracy rate (Correct), speech detection error (SE), and non-speech detection error (NE) are displayed on the upper or lower right of each subfigure. The total misclassification error was reduced by $61.4 \%$ (-10 dB), 74.5\% (-5 dB), 79.0\% (0 dB), 84.3\% (5 dB), and $82.9 \%(10 \mathrm{~dB})$ when the window length $R$ was increased from 2 to 30 frames. The percentage is the ratio between the reduced misclassification error (when $R$ was changed from 2 to 30 frames) and the misclassification error when $R$ was 2 .

The distributions of the LSFM feature for all 12 kinds of noises at $0-\mathrm{dB}$ SNR were investigated. $M$ is fixed to be 10 , and $R$ is chosen to be 30 . The discriminative power of this LSFM feature can be measured by the separateness of its distribution for speech and non-speech. As shown in Figures 6, 7, and 8, there is overlap between the histograms of $\log _{10}\left(L_{S+N}\right)$ and $\log _{10}\left(L_{N}\right)$. We calculated the total misclassification error which is the sum of the speech detection error and non-speech detection error. From the figures, we can conclude that for most noises considered
(9 out of 12 kinds of noises), the proposed LSFM feature resulted in a misclassification error smaller than $10 \%$ : white $(7.86 \%)$, pink $(7.75 \%)$, tank $(7.47 \%)$, military vehicle $(7.75 \%)$, jet cockpit (9.32\%), HF channel (8.30\%), F-16 cockpit $(8.89 \%)$, car interior $(8.14 \%)$, and speech shaped (7.84\%). For factory floor (25.86\%), machine gun (45.42\%), and speech babble (24.08\%), the misclassification errors were comparatively high. The factory floor is that of cutting noise, that of the machine gun is impulsive in nature, and that of speech babble is speechlike. One possible reason for the poor performance is the mismatch of $M$ and $R$.

\section{The proposed LSFM-based VAD algorithm}

The proposed VAD algorithm assumes that the signal spectrum is more organized during speech segments than during noise segments [18]. It adopts the average spectrum over a long-term window instead of instantaneous values of the spectrum. Typically, a periodogram is 


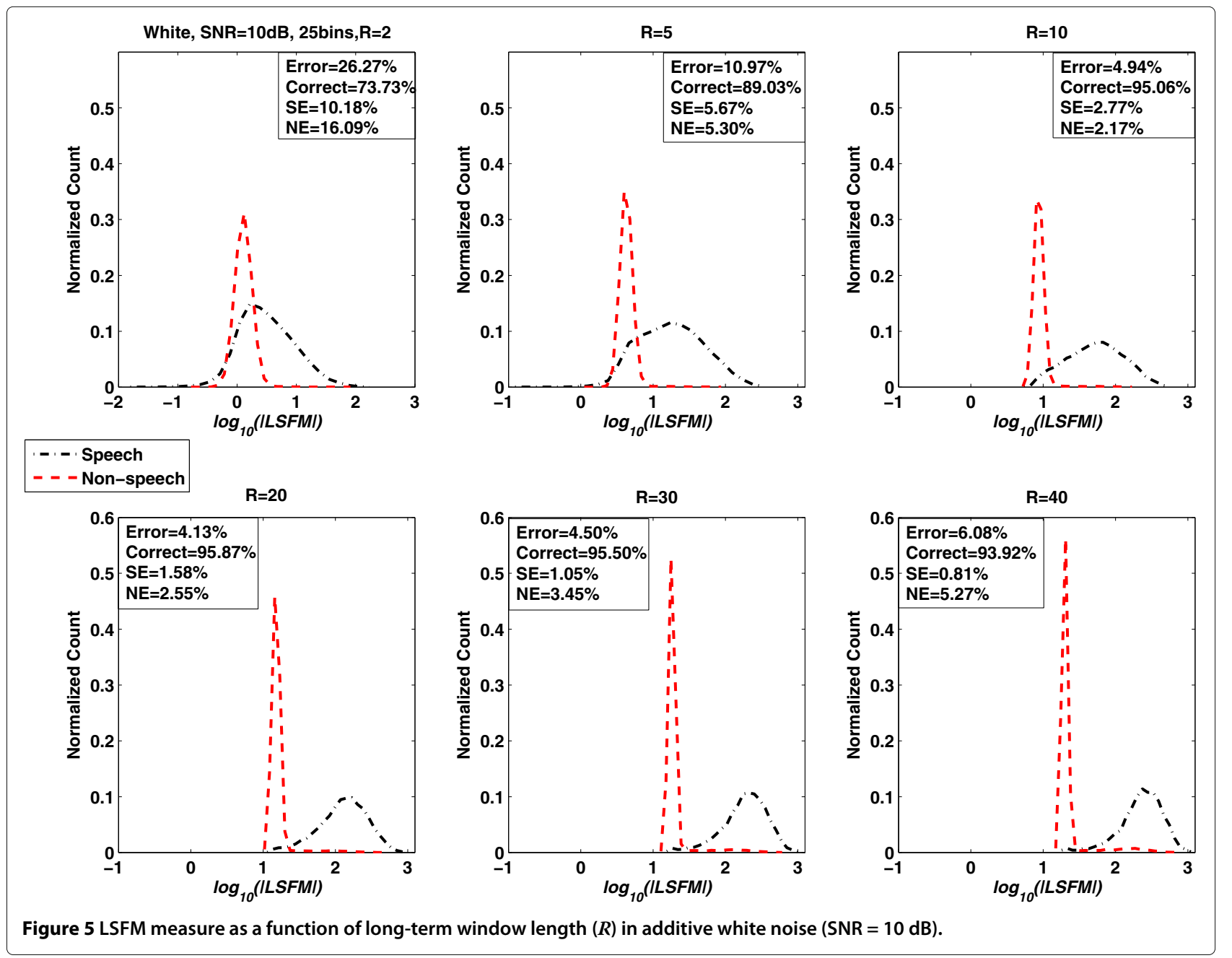

commonly employed for spectrum estimation, but it is well known that the periodogram is an inconsistent spectral estimator. According to [8], the Welch-Bartlett method [19] was found to give a good trade-off between variance reduction and spectral resolution reduction. Therefore, in our proposed algorithm, the signal spectrum is estimated using the Welch-Bartlett method.

A block diagram of the proposed LSFM-based VAD algorithm is shown in Figure 9. The algorithm can be described as follows. The input speech signal is decomposed into frames of $20 \mathrm{~ms}$ in length with an overlap of $10 \mathrm{~ms}$ by the Hann window. The spectrum of the segmented signal is estimated using the Welch-Bartlett method. At the $m$ th frame, the LSFM feature $L_{x}(m)$ is computed using the previous $R$ frames. The initial decision about whether there contains speech in the last $R$ frames is made through the comparison with an adaptive threshold. The initial decision is denoted by $V_{\mathrm{INL}}$. If there is a speech frame existing over the previous $R$ frames ending at the $m$ th frame, $V_{\mathrm{INL}}(m)=1$; otherwise,
$V_{\mathrm{INL}}(m)=0$ and there are only non-speech frames over the previous $R$ frames. We adopt the voting scheme proposed by Ghosh et al. [15] to make the final VAD decisions on a $10-m s$ interval. First, the initial decisions, $V_{\mathrm{INL}}(m)$, $V_{\mathrm{INL}}(m+1), \ldots, V_{\mathrm{INL}}(m+R-1)$, are collected for those long windows which overlap with the target 10-ms interval. Then, the target 10-ms interval is marked to be speech if there is $80 \%$ or above of those initial decisions that contain speech; otherwise, it is marked as non-speech. The $80 \%$ was gotten empirically, which provided the maximum VAD accuracy for most noises tested over five SNR levels.

In general, speech is a low-pass signal, and the frequency range of $500 \mathrm{~Hz}$ to $4 \mathrm{kHz}$ is crucial for speech intelligibility [20]. Hence, for a better discrimination, the start bin, $k_{s}$, and the end frequency bin, $k_{e}$, are calculated by:

$$
k_{s}=N_{\mathrm{DFT}}\left(\frac{500}{f_{s}}\right)
$$




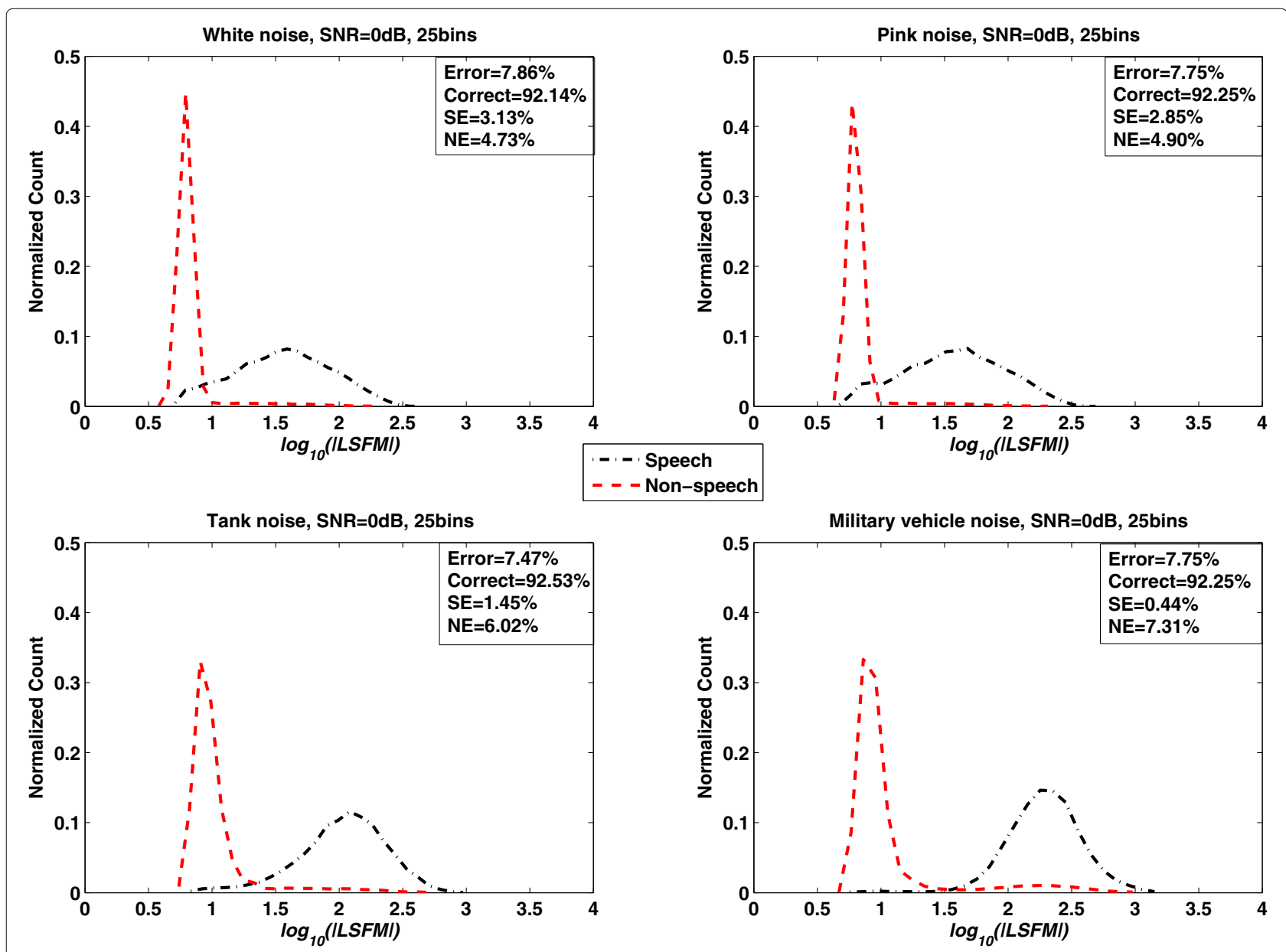

Figure 6 Histogram of the logarithmic LSFM measure for white, pink, tank, and military vehicle noises (SNR $=0 \mathrm{~dB})$. Upper left: white noise, upper right: pink noise, lower left: tank noise, and lower right: military vehicle noise.

$$
k_{e}=N_{\mathrm{DFT}}\left(\frac{4,000}{f_{s}}\right)
$$

in which $f_{s}$ is the sampling frequency and $N_{\mathrm{DFT}}$ is the order of Discrete Fourier Transform (DFT) which is used to calculate the spectral estimate of the observed signal. In our experiment, $f_{s}=16 \mathrm{kHz}$ and $N_{\mathrm{DFT}}=512$. The frequencies, $\omega_{k}$, are uniformly distributed between $500 \mathrm{~Hz}$ and $4 \mathrm{kHz}$.

An illustrative example of the VAD output is shown in Figure 10. A high spectral flatness indicates that the spectrum has a similar amount of power in all spectral bands, which would sound similar to white noise, and the graph of the spectrum would appear relatively flat and smooth. A low spectral flatness indicates that the spectral power is concentrated in a relatively small number of bands; this means that the spectrum is more organized, and the graph of the spectrum would appear 'spiky'. Hence, the spectral flatness measure is a good feature for VAD.

\subsection{Selection of $M$ and $R$}

$M$ and $R$ are parameters used for computing the LSFM feature $L_{x}$. We want to choose the appropriate $M$ and $R$ so that the separateness of the distribution for noise and speech is maximized since the more it is separated, the better the final VAD decision is. The total misclassification errors (sum of speech detection error and non-speech detection error) for all combinations of $M(1,5,10,20,30$, and 40$)$ and $R(5,10,20,30$, and 40) are computed over 12 types of noise for five SNR levels $(-10,-5,0,5$, and $10 \mathrm{~dB}$ ). The test speech set is the same with the one we used for the demonstration of the discriminative power of the proposed LSFM feature in Section 2.2.

The total misclassification error as a function of different combinations of $M$ and $R$ is shown in Figures 11, 12, 13, and 14. The best combination of $M$ and $R$ for each noise at each SNR level is written on the upper or lower right of each subfigure. After the summed up frequency of each $M$ and $R$ that appeared in the subfigures, we conclude that $(10,30)$ is the optimal combination 


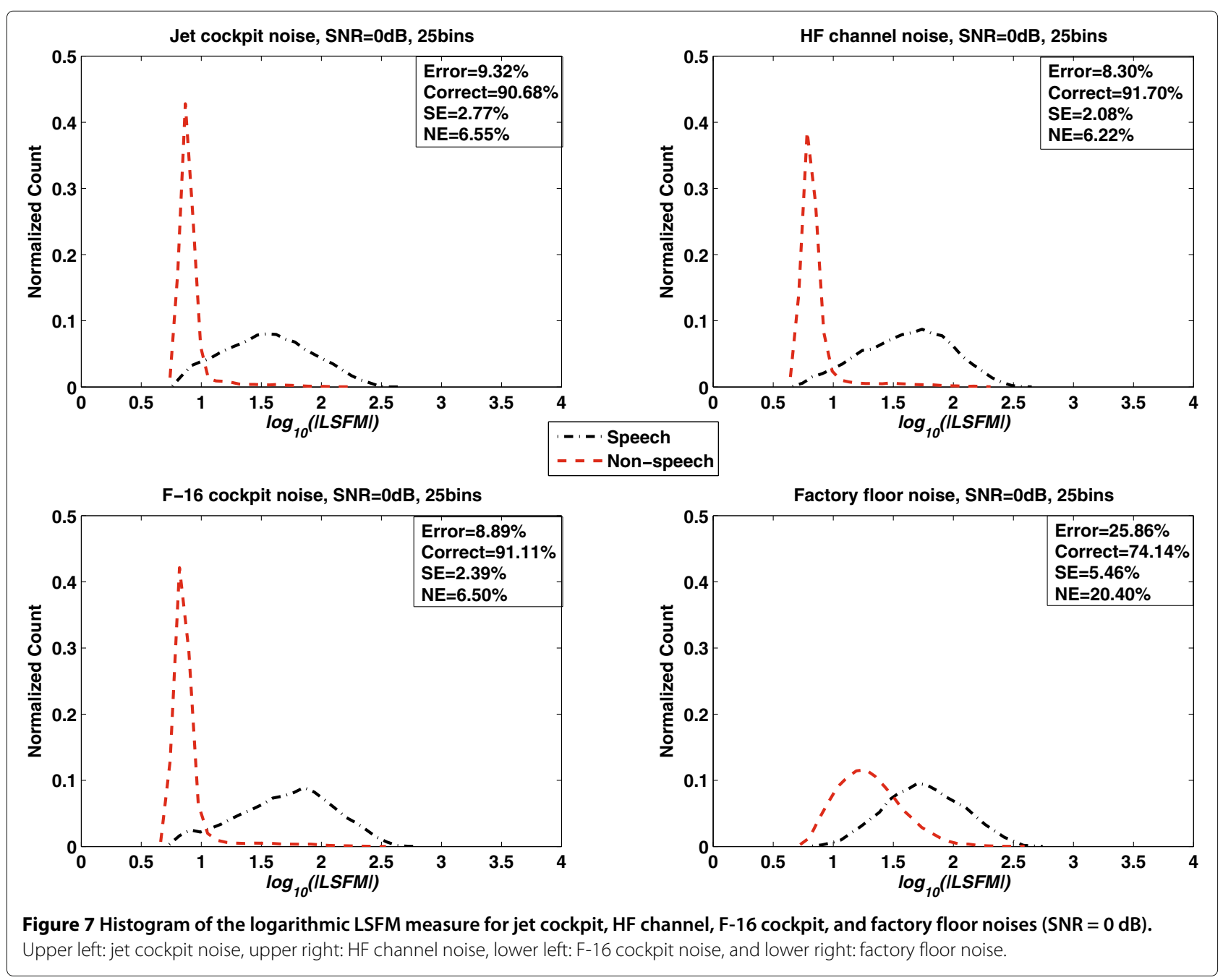

which appeared most frequently ( $M=10$ appeared 25 times out of the 60 subfigures in total; $R=30$ also appeared 25 times out of 60 subfigures in total). This fixed combination $(10,30)$ is then adopted for all the following tests.

Furthermore, from Figures 11, 12, 13, and 14, we observe that for the same $R$ value, if $M$ is increased from 1 to 10 , the total misclassification error is decreased for most cases tested. However, when $M$ is larger than 10 , even if $M$ is increased, the total misclassification error stops decreasing any further. This observation verified the choice of 10 to be the optimal value of $M$.

It is also worth mentioning that for those noise types and SNR levels whose optimal $M$ and $R$ combination is not $(10,30)$, the fixed combination $(10,30)$ still works well. Table 1 shows the points that the total misclassification error of adopting the fixed combination $(10,30)$ is worse (higher error value) than utilizing the best combination of $M$ and $R$ for each noise type and SNR level shown in the subfigures. Except for cutting factory floor noise and impulsive machine gun noise, the differences are all less than five points.

For machine gun noise, the optimal choice of $R$ is 5 for all SNR levels. Machine gun noise is an impulsive noise which consists of two types of sounds, namely gunshot and silence between gunshots [15]. When $R$ is 30, the long analysis window would include both types of sounds. Therefore, the spectral power over these 30 frames will be less uniform; the LSFM feature value will then be small, and there will be more classification errors compared to the case when $R$ is 5 . Similarly, for factory floor noise, the optimal choice of $M$ is 1 for all SNR levels. Factory floor noise [21] was recorded near plate-cutting and electrical welding equipment which shows a repetitive pattern. According to [19], the variance of estimated power spectrum will not be obviously reduced if the overlapped frames are highly correlated with each other. Therefore, averaging over $M$ overlapped frames will cause 


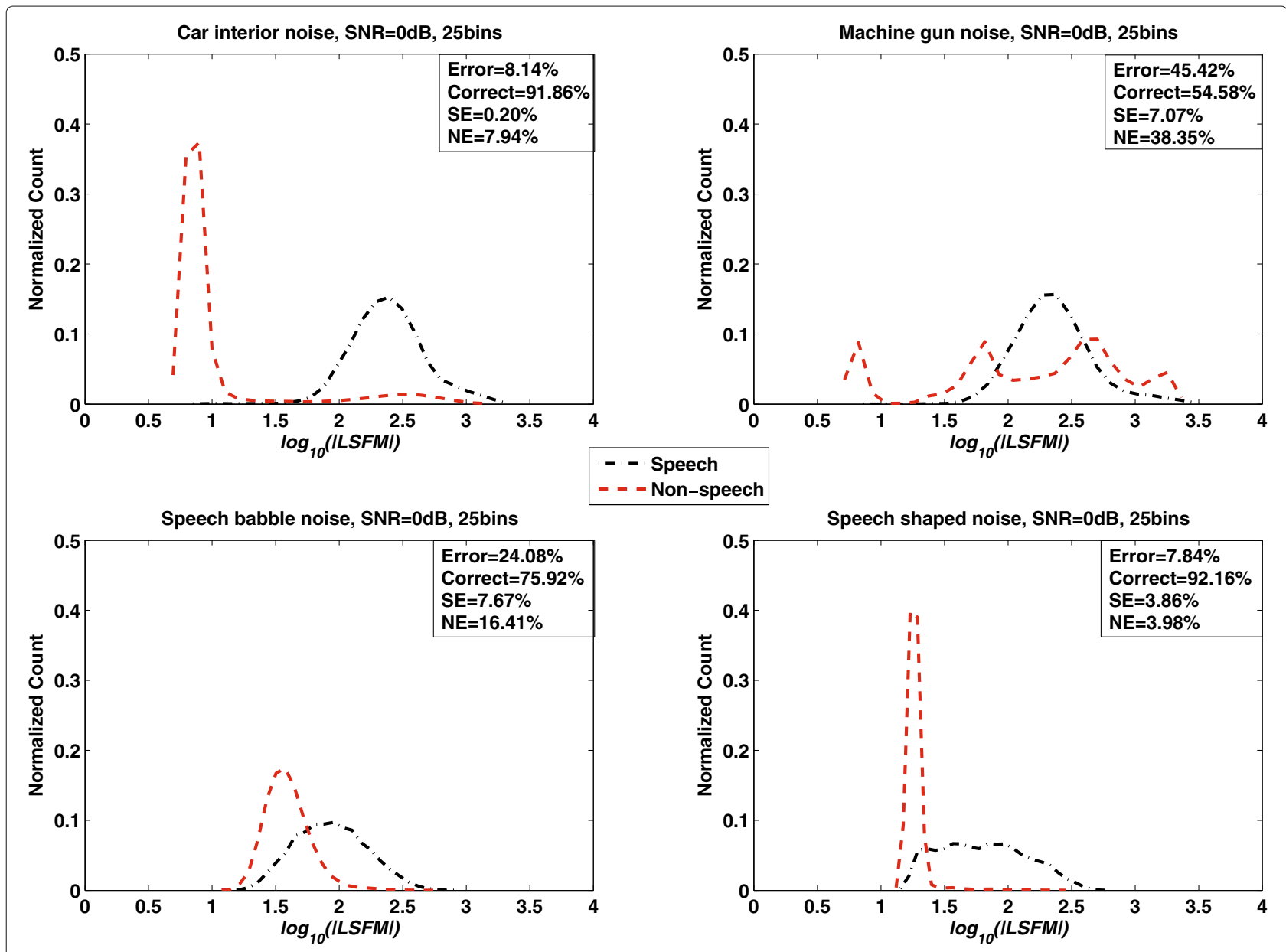

Figure 8 Histogram of the logarithmic LSFM measure for car interior, machine gun, speech babble, and speech-shaped noises (SNR = $\mathbf{0} \mathbf{d B}$ ). Upper left: car interior noise, upper right: machine gun noise, lower left: speech babble noise, and lower right: speech-shaped noise.

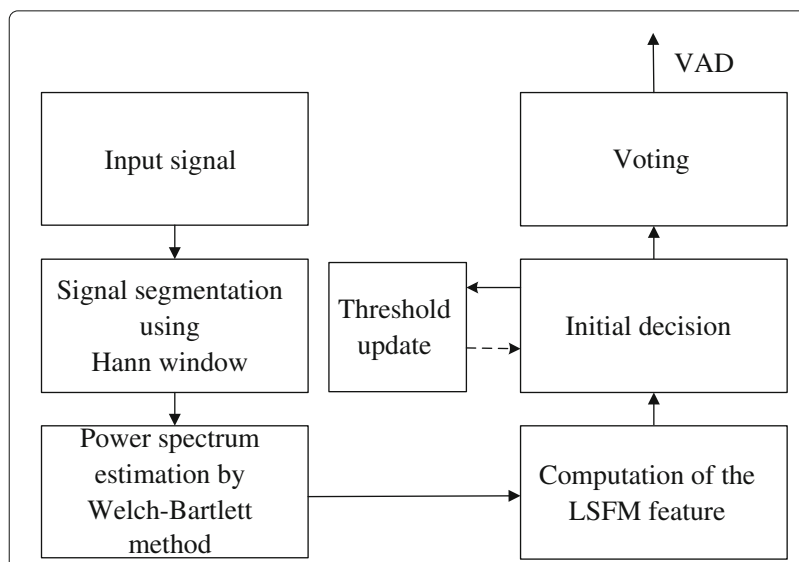

Figure 9 Block diagram of the proposed LSFM-based VAD algorithm. more misclassification errors compared to the case when $M$ is 1 .

\subsection{Adaptive threshold}

Unlike $M$ and $R$, a fixed threshold would lose its efficiency when facing varying acoustic environments. Therefore, it is more suitable to design an adaptive threshold [22]. From Equations 2 to 4 , we can conclude that $(R+M-1)$ frame $(0.39 \mathrm{~s}$ for fixed $R=30$ and $M=10)$ information is needed to acquire the first LSFM feature value. In our implementations, the initial $1.39 \mathrm{~s}$ of the test signal $x(n)$ is always assumed to be non-speech. From this $1.39 \mathrm{~s}$ of $x(n)$, 100 realizations of $L_{N}$ can be collected and saved to $\psi_{\mathrm{INL}}$.

The threshold is initialized to be

$$
\mathrm{THR}_{\mathrm{INL}}=\min \left(\psi_{\mathrm{INL}}\right) .
$$

To update the threshold at the $m$ th frame, we used two buffers $\psi_{S}+N$ and $\psi_{N} \cdot \psi_{S}+N$ stores the LSFM measures of the last 100 long window ending at the $m$ th frame which was decided as containing speech; similarly, $\psi_{N}$ stores 


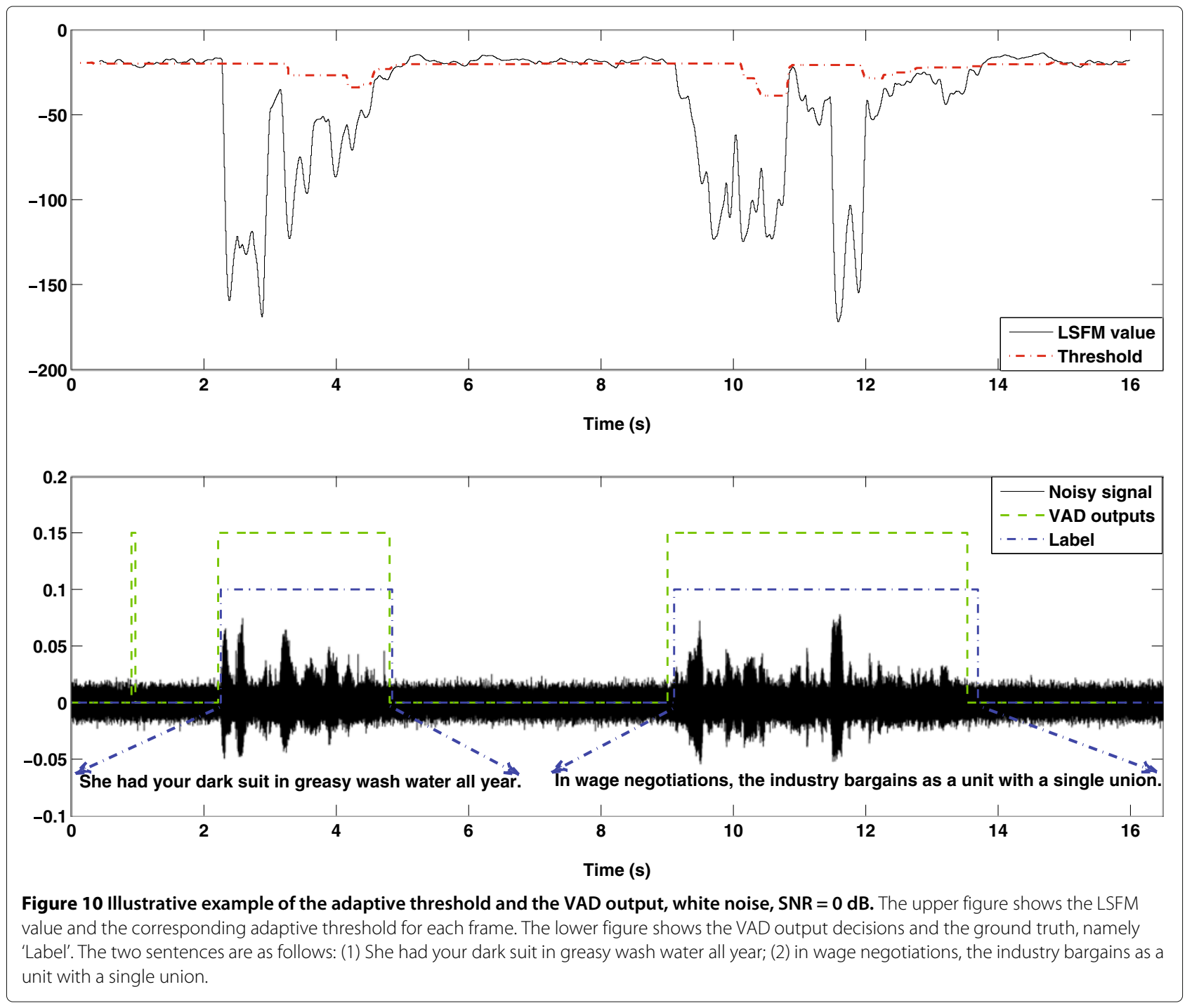

the LSFM measures of the last 100 long window ending at the $m$ th frame which was decided as including nonspeech information only. The adaptive threshold for the $m$ th frame is then updated as:

$$
\operatorname{THR}(m)=\lambda \times \min \left(\psi_{S+N}\right)+(1-\lambda) \times \max \left(\psi_{N}\right),
$$

where $\lambda$ is the parameter of the convex combination. We experimentally found that $\lambda=0.55$ results in the maximum accuracy rate in VAD decisions over the TIMIT training set.

\section{Evaluation setup}

The proposed VAD algorithm was trained and tested using a speech database that is phonetically balanced. The system was evaluated using the error rate and accuracy rate metrics.

\subsection{Database description}

For the evaluation of VAD algorithms, TIMIT corpus is preferred since it provides manual transcription down to word and phoneme levels. The reference labels are computed using the start and end times of the utterance obtained from the TIMIT transcription (.phn files). Some experiments are carried out on the core TIMIT test set consisting of 24 individual speakers ( 16 males, 8 females) of eight different dialects, each speaking 10 phonetically balanced English sentences. The utterances of TIMIT corpus are short (about $3.5 \mathrm{~s}$ ), and around $90 \%$ of which are speech; this may introduce a bias when comparing the distributions of speech and non-speech. To reduce this effect and make it closer to real-world scenarios, 2-s silence was added before and after each utterance to simulate a typical telephone conversation $[8,15,23]$ in which the ratio of speech to non-speech is almost $40 \%$ to $60 \%$. 


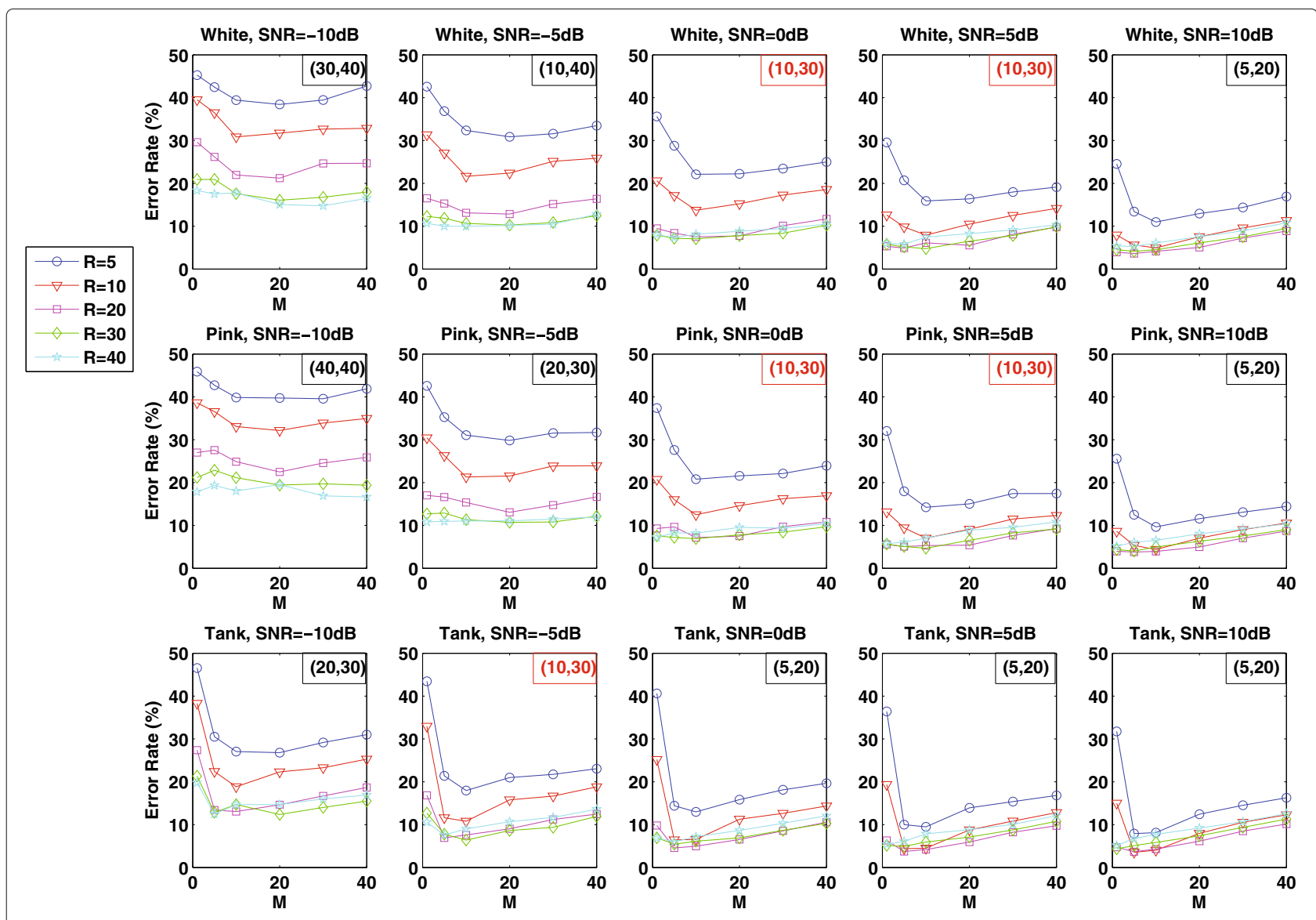

Figure 11 Total misclassification error as a function of $\boldsymbol{M}$ and $\boldsymbol{R}$ combination for white, pink, and tank noises. Upper row: white noise, middle row: pink noise, and lower row: tank noise. SNR $=-10,-5,0,5$, and $10 \mathrm{~dB}$.

The noise of 11 categories taken from the NOISEX-92 database [21] and speech-shaped noise are added at five different SNR levels $(-10,-5,0,5$, and $10 \mathrm{~dB})$ to the signal concatenated by all 240 sentences. The noise samples from the NOISEX-92 database are resampled to $16 \mathrm{kHZ}$ according to the experiment requirement. Among the 12 kinds of noises, white noise and pink noise are stationary noises while others are all non-stationary noises, namely tank, military vehicle, jet cockpit, HF channel, F-16 cockpit, factory floor, car interior, machine gun, speech babble, and speech-shaped noises. The test set for each noise and SNR thus consisted of $28.10 \mathrm{~min}$ of noisy speech of which $62.51 \%$ was only noise.

\subsection{Performance evaluation}

Performance of a VAD algorithm can be evaluated both subjectively and objectively. In general, subjective evaluation is done through a listening test, and VAD decision errors are detected based on human perception [24]. On the other hand, objective evaluation relies on a mathematical criterion for judging. However, subjective listening tests like ABC [24] fail to consider the effect of the false alarm which is inappropriate for a thorough evaluation of a VAD algorithm [8]. Therefore, the objective evaluation scheme proposed by Freeman et al. [2] was adopted to evaluate the performance of the proposed VAD algorithm.

\subsubsection{Error rate}

The four traditional parameters that describe the error rate are as follows:

- Front-end clipping (FEC). Clipping introduced in passing from noise to speech activity.

- Mid-speech clipping (MSC). Clipping due to speech misclassified as noise in an utterance.

- Noise detected as speech (NDS). Noise detected as speech within a silence period.

- Carry over (OVER). Noise interpreted as speech due to the VAD flag remaining active in passing from speech activity to noise.

These four parameters are illustrated in Figure 15. Among them, FEC and MSC are indicators of true 


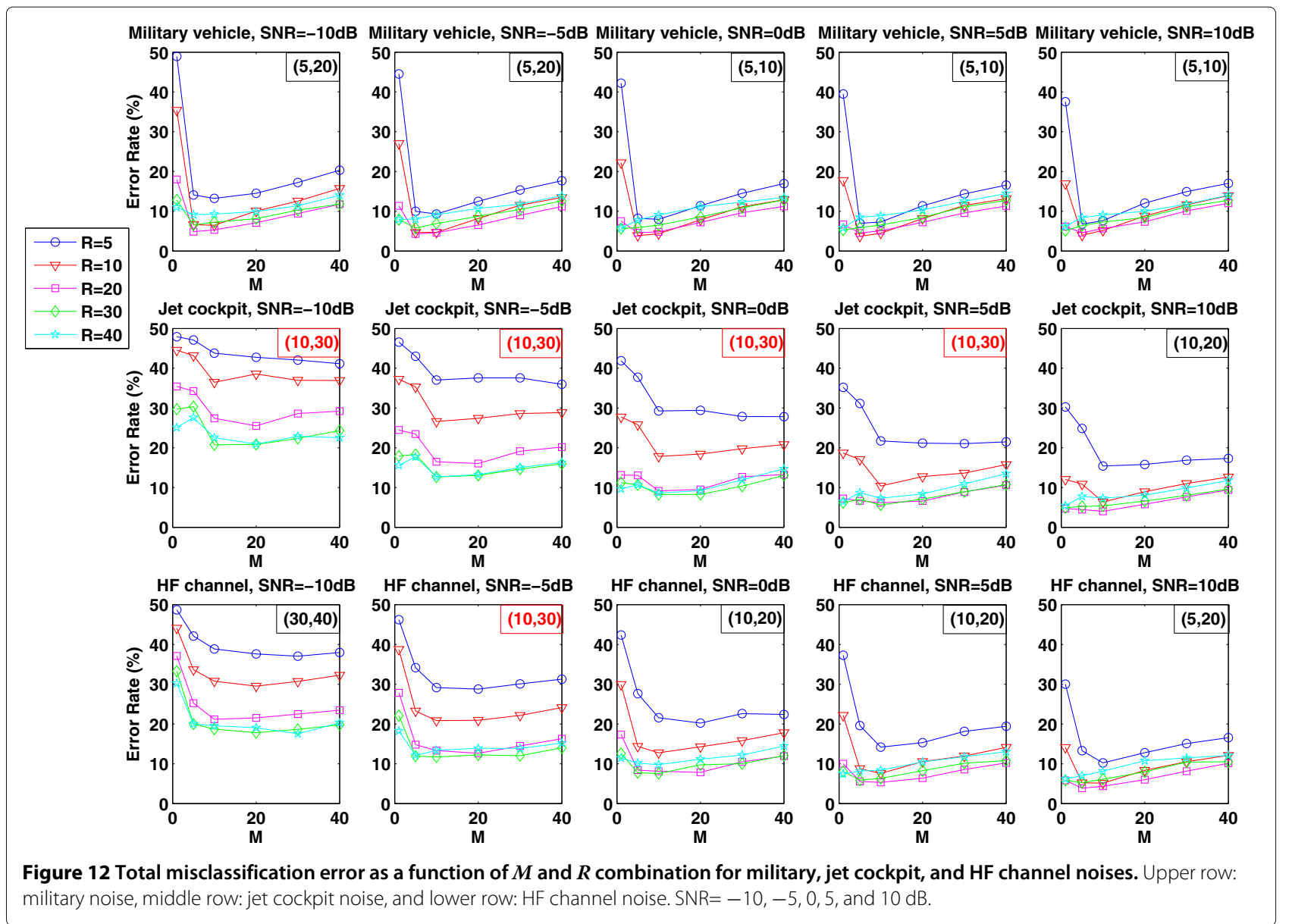

rejection, while NDS and OVER are indicators of false acceptance. Thus, in order to obtain the best overall system performance, all four parameters should be minimized.

\subsubsection{Accuracy rate}

Although the method described above provides useful objective information concerning the performance of a VAD algorithm, it only gives the error rate of the system. Parameters which describe the accuracy rate are needed for a thorough analysis of the detection results. Three parameters concerning the accuracy rate are described as follows:

- CORRECT. They are correct decisions made by VAD algorithm.

- Speech hit rate (HR1). Speech frames that are correctly detected among all speech frames.

- Non-speech hit rate (HRO). Non-speech frames that are correctly detected among all non-speech frames.

Among the three parameters, HR1 and HR0 define the fraction of all actual speech frames or non-speech frames that are correctly detected as speech frames or non-speech frames, respectively [12]. The speech hit rate and non-speech hit rate are calculated as follows:

$$
\mathrm{HR} 1=\frac{N_{1,1}}{N_{1}^{\mathrm{ref}}} \quad \mathrm{HR} 0=\frac{N_{0,0}}{N_{0}^{\mathrm{ref}}},
$$

where $N_{1}^{\text {ref }}$ and $N_{0}^{\text {ref }}$ are the numbers of real speech and non-speech frames in the whole database, respectively, while $N_{1,1}$ and $N_{0,0}$ are the numbers of speech and nonspeech frames correctly classified. The overall accuracy rate (CORRECT) is then defined as:

$$
\text { CORRECT }=\frac{N_{1,1}+N_{0,0}}{N_{1}^{\mathrm{ref}}+N_{0}^{\mathrm{ref}}} .
$$

All three parameters should be maximized to get the best performance.

\section{Simulation results}

In order to gain a comparative analysis of the proposed LSFM-based VAD performance, three modern standardized VAD schemes and one recent long-term algorithm, namely ETSI adaptive multi-rate (AMR) VAD options 1 and 2 (AMR1 and AMR2) [25], the G.729B VAD [26], and LTSV, were also evaluated. The implementations of these 


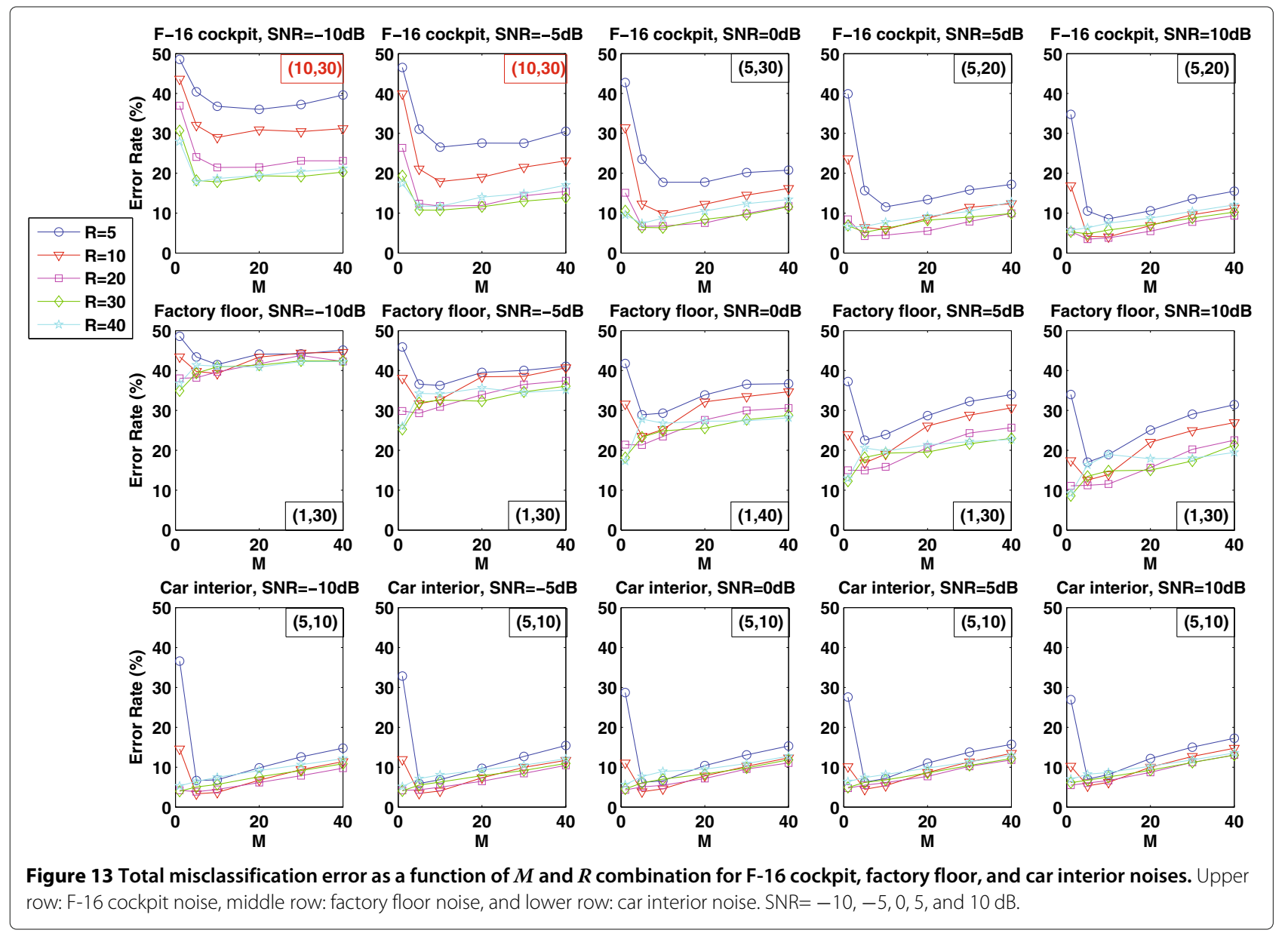

three schemes were taken from the authors' $\mathrm{C}$ implementations $[27,28]$, respectively.

One important aspect of the comparison is the different frame lengths used. The proposed schemes, the G.729B VAD and LTSV-based VAD, produce a decision every $10 \mathrm{~ms}$, while the AMR VADs need $20 \mathrm{~ms}$. In order to be comparable, the frame-wise VAD decisions produced by the AMR VADs were compared to a set of reference labels generated every $20 \mathrm{~ms}$ from the TIMIT phonetic level transcription. Meanwhile, the proposed schemes, the G.729B VAD and LTSV-based VAD, were compared to a set of reference labels generated every $10 \mathrm{~ms}$ from the TIMIT phonetic level transcription. The TIMIT utterances were down-sampled to $8 \mathrm{kHz}$ for the software implementations of the G.729B VAD and AMR VADs. The final VAD decisions were made, and the accuracy rate and error rate were computed for 12 noises and five SNRs.

\subsection{Performance average over all twelve kinds of noises}

In Figure 16, the proposed LSFM-based VAD is compared with three standards and LTSV-based VAD in terms of accuracy rate and error rate for SNR levels ranging from -10 to $10 \mathrm{~dB}$. Note that the results in Figure 16 are averaged values for all 12 noises. The first row of the figure shows the accuracy rates which include CORRECT, HR1, and HR0. The behavior of the different VADs is analyzed. G.729B suffers poor CORRECT(62.74\% at $-10 \mathrm{~dB})$ and HR1 $(33.62 \%$ at $-10 \mathrm{~dB})$ with the increasing noise level, while it keeps a steady and relatively high HR0 for the whole range of SNRs ( $80.33 \%$ on average). AMR1 performs much better than G.729B for both CORRECT and HR1 while suffering degradation of HR0 when the SNR level is increased. AMR2 improves considerably over AMR1 in CORRECT mainly because of the high HR0 over all SNRs (88.96\% on average) while yielding similar HR1 with AMR1. LTSV performs very well under low SNR conditions (80.73\% CORRECT at $-10 \mathrm{~dB}$ ) but becomes saturated (around 91\% since $5 \mathrm{~dB}$ ) at higher SNRs. Our proposed LSFM-based VAD yields the best CORRECT for all SNRs and shows a steady improvement with the increased SNR.

Similarly, the second row of Figure 16 shows the error rates which include FEC, MSC, OVER, and NDS. G.729B performs the worst on average in terms of true rejection rate (FEC and MSC). However, the OVER of G.729B is the lowest among the five VADs tested. AMR1 and 


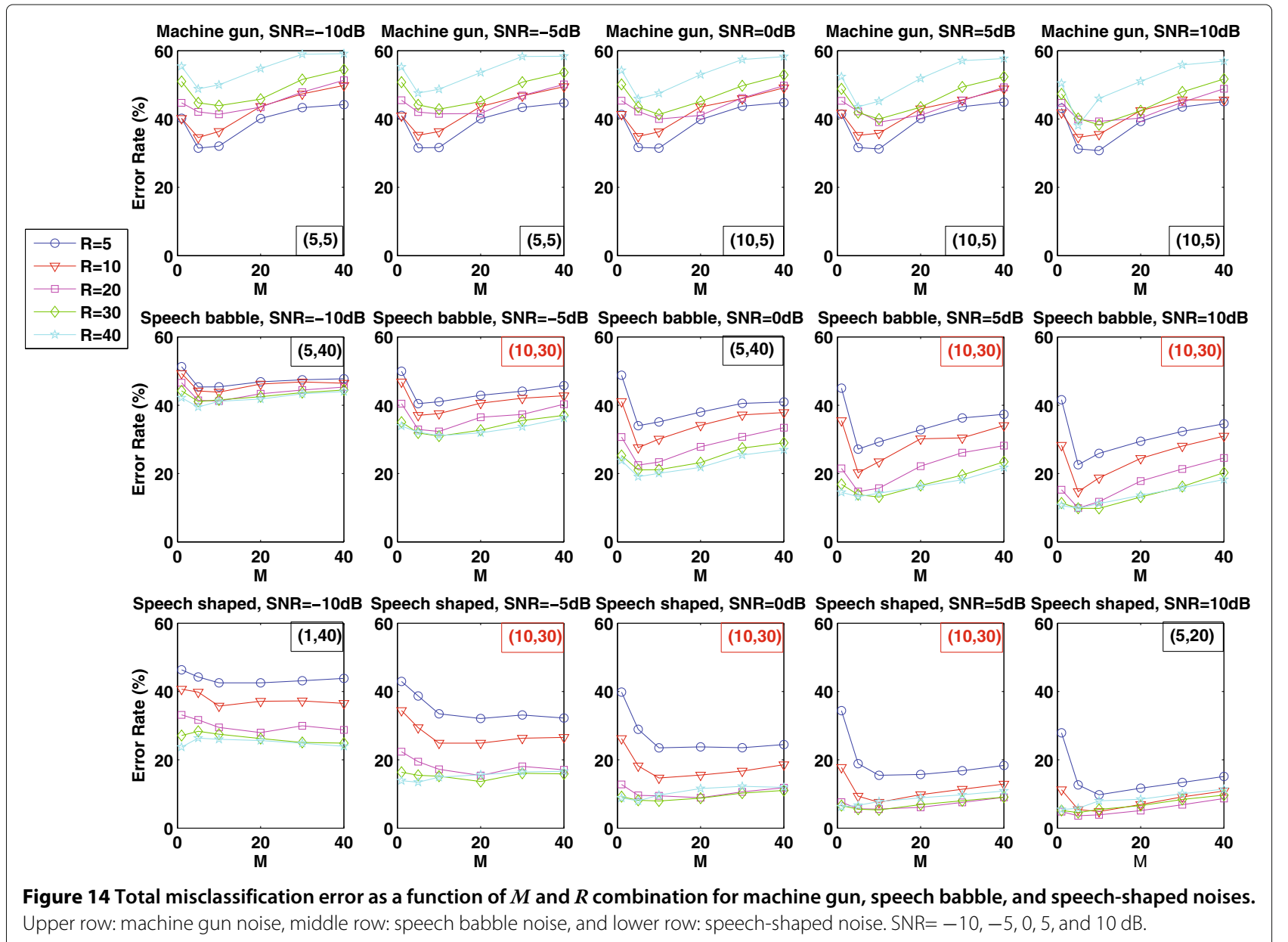

Table 1 Total misclassification error difference between adopting the fixed combination $(10,30)$ and utilizing the best $(M, R)$ combination

\begin{tabular}{lccccc}
\hline Noise type & $\mathbf{- 1 0} \mathbf{~ d B}$ & $\mathbf{- 5} \mathbf{d B}$ & $\mathbf{0 ~ d B}$ & $\mathbf{5 ~} \mathbf{~ B}$ & $\mathbf{1 0} \mathbf{~ B}$ \\
\hline White & 2.85 & 0.69 & 0 & 0 & 0.87 \\
Pink & 4.49 & 0.64 & 0 & 0 & 1.19 \\
Tank & 2.32 & 0 & 1.59 & 2.20 & 2.17 \\
Military vehicle & 2.36 & 2.59 & 2.62 & 2.82 & 3.31 \\
Jet cockpit & 0 & 0 & 0 & 0 & 1.36 \\
HF channel & 1.15 & 0 & 0.57 & 1.02 & 2.27 \\
F-16 cockpit & 0 & 0 & 0.10 & 1.94 & 2.30 \\
Factory floor & 6.04 & 7.35 & 7.59 & 7.04 & 6.25 \\
Car interior & 2.33 & 2.82 & 3.16 & 2.33 & 2.21 \\
Machine gun & 12.45 & 11.33 & 9.83 & 8.74 & 7.56 \\
Speech babble & 2.11 & 0 & 2.068 & 0 & 0 \\
Speech shaped & 3.80 & 0 & 0 & 0 & 1.84 \\
\hline
\end{tabular}

The numbers are the points that worse (higher error value) than utilizing the best $(M, R)$ combination for each noise type and SNR level shown in the subfigures.
AMR2 yield similar true rejection rates for all tested SNRs, while AMR2 gives smaller false acceptance rate (NDS and OVER) especially for NDS (around four points less than AMR1 for all SNRs). LTSV leads to the lowest true rejection rate, while LSFM achieved the best performance in terms of NDS. The proposed LSFM-based VAD acquires a comparatively higher FEC in low SNRs (smaller than $-5 \mathrm{~dB}$ ) because of the averaging property of this algorithm shown in Equations 2, 3, and 4.

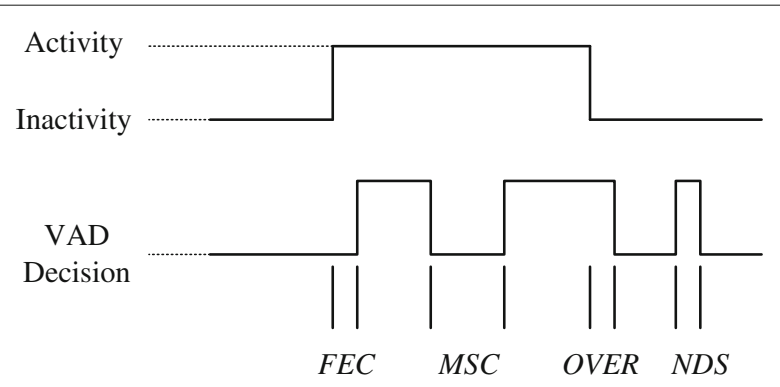

Figure 15 Objective parameters for performance evaluation. 

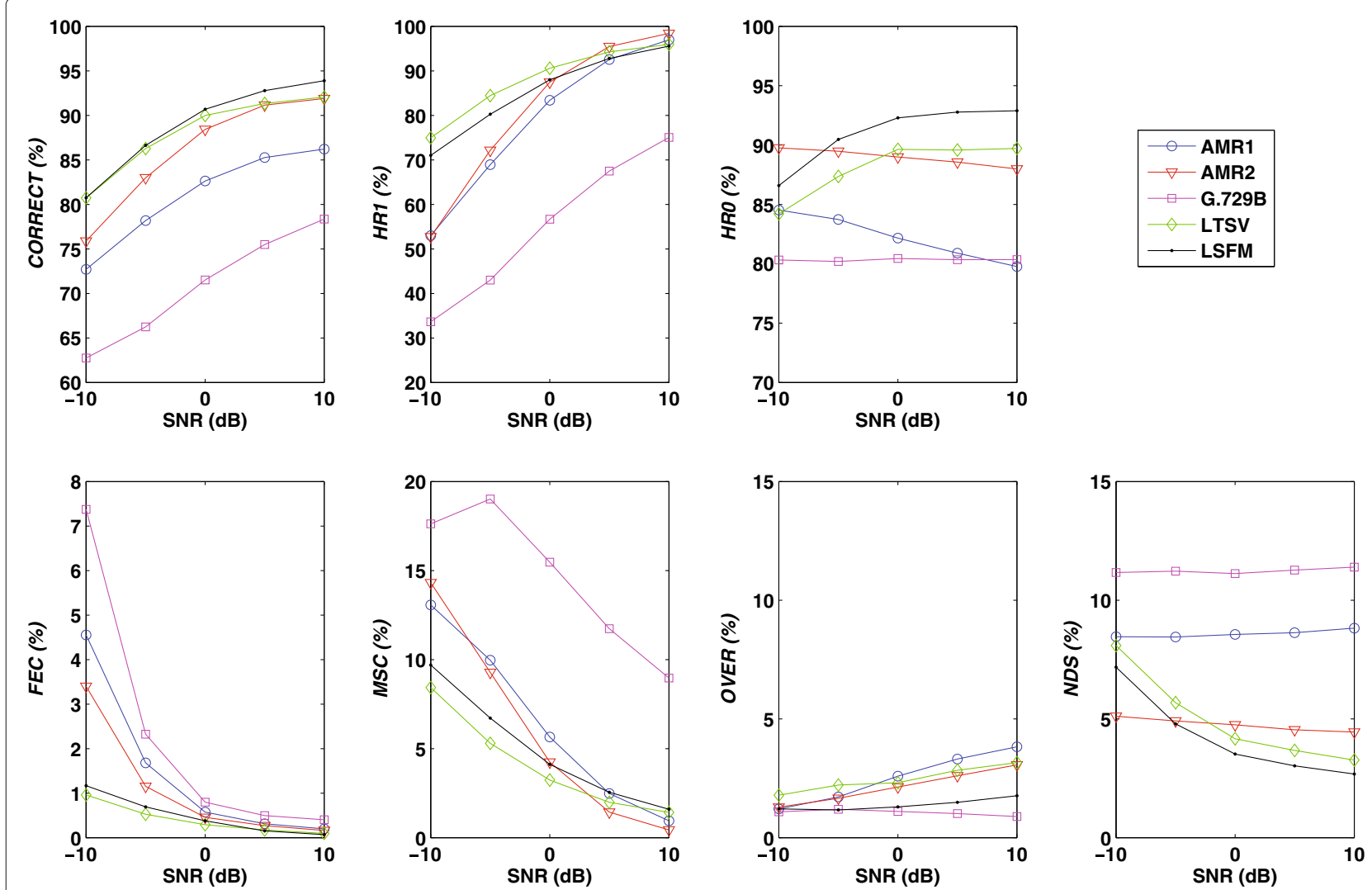

Figure 16 Accuracy and error rate comparisons for five VAD schemes averaged over 12 noises for five SNRs. Accuracy rate: CORRECT, HR1, and HRO; error rate: FEC, MSC, OVER, and NDS. Five VAD schemes: AMR1, AMR2, G.729B, LTSV, and LSFM. Five SNRs ( $-10,-5,0,5$, and 10 dB).

Table 2 summarizes the results provided by LSFMbased VAD over the different VAD methods being evaluated by comparing them in terms of the average accuracy rate and error rate for all 12 noises over five SNR levels ranging from -10 to $10 \mathrm{~dB}$. LSFM achieves the best CORRECT (88.95\%) and HR0 (91.00\%), while LTSV yields the best HR1 (88.04\%).

\subsection{Performance average over five SNRs}

Figure 17 shows the three different accuracy rate evaluation metrics averaged over five SNRs for 12 kinds of

Table 2 Average performance comparison for all 12 noises over five SNR levels ranging from -10 to $10 \mathrm{~dB}$

\begin{tabular}{lccccc}
\hline VAD & AMR1 & AMR2 & G.729B & LTSV & LSFM \\
\hline CORRECT & 81.00 & 86.07 & 70.87 & 88.08 & 88.95 \\
HR1 & 78.96 & 81.25 & 55.16 & 88.04 & 85.53 \\
HRO & 82.22 & 88.96 & 80.33 & 88.10 & 91.00 \\
FEC & 1.46 & 1.09 & 2.28 & 0.41 & 0.49 \\
MSC & 6.42 & 5.94 & 14.56 & 4.07 & 4.93 \\
OVER & 2.53 & 2.15 & 1.06 & 2.46 & 1.39 \\
NDS & 8.59 & 4.75 & 11.24 & 4.98 & 4.24 \\
\hline
\end{tabular}

noises computed for AMR1, AMR2, G.729B, LTSV, and LSFM-based VAD algorithms. From Figure 17, it is clear that in terms of CORRECT, LTSV is the best among all four reference VAD algorithms considered here. Hence, the proposed LSFM-based VAD is compared with the LTSV-based VAD. We observe that on average, the LSFMbased VAD is better than the LTSV-based VAD in terms of CORRECT for tank $(0.52 \%)$, military vehicle $(1.40 \%)$, F-16 cockpit $(0.34 \%)$, car interior $(2.12 \%)$, machine gun (1.88\%), and speech babble (7.63\%) noises, and it is worse for white $(1.10 \%)$, pink $(0.79 \%)$, jet cockpit $(0.64 \%)$, HF channel $(0.15 \%)$, factory floor $(0.15 \%)$, and speech-shaped $(0.58 \%)$ noises. The number in the bracket indicates the absolute CORRECT by which the proposed LSFM-based VAD is better or worse than the LTSV-based VAD. The mean CORRECT over all 12 noise types of our proposed LSFM-based VAD is $0.87 \%$ higher than that of the LTSVbased VAD. Furthermore, the proposed LSFM-based VAD outperforms LTSV-based VAD in terms of HR0 over most noises (11 out of 12) that were considered.

Figure 18 shows the four different error rate evaluation metrics (FEC, MSC, OVER, and NDS), averaged over five SNRs for 12 kinds of noises, computed for AMR1, AMR2, G.729B, LTSV, and LSFM algorithms. From Figure 18, it 


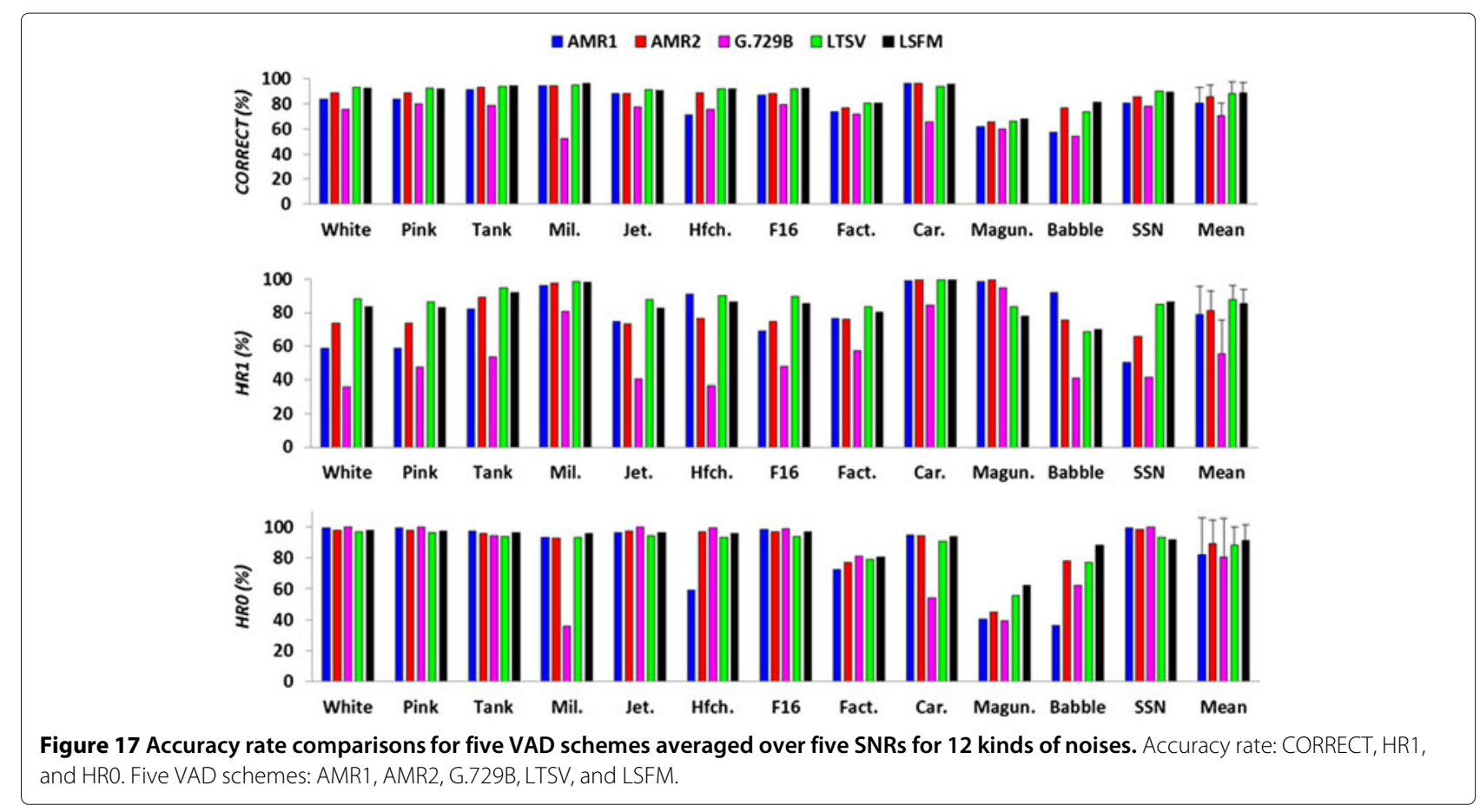

is clear that the performance of LSFM-based VAD outperforms the LTSV-based VAD in terms of OVER (all 12 noises) and NDS (9 out of 12). For example, The proposed LSFM-based VAD has a smaller NDS score for tank $(0.26 \%)$, military vehicle $(0.10 \%)$, jet cockpit $(0.35 \%)$, HF channel(0.44\%), F16 cockpit (0.80\%), factory floor $(0.42 \%)$, car interior $(0.31 \%)$, machine gun $(2.15 \%)$, and babble (5.83\%) noises. The number in the bracket indicates the absolute NDS by which the proposed LSFM-based VAD is smaller than the LTSV-based VAD. Moreover, values of
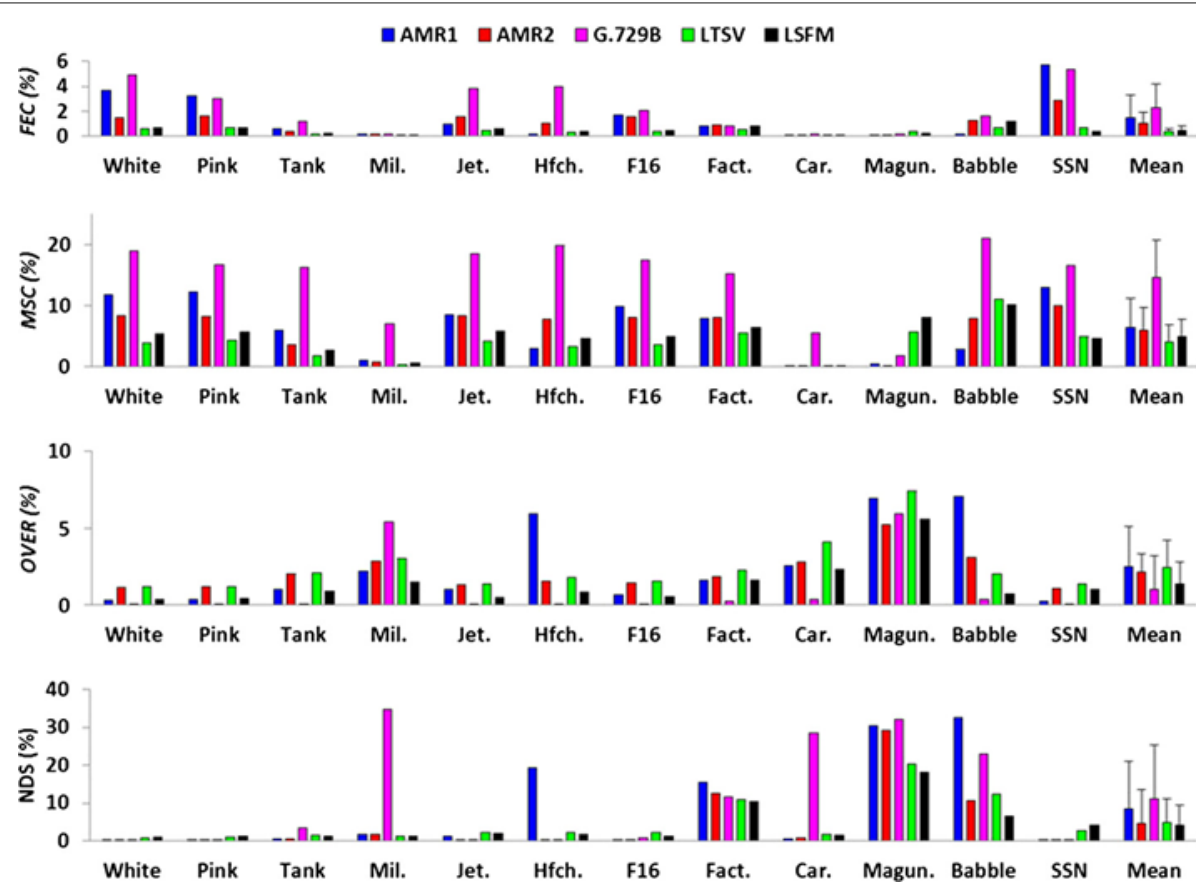

Figure 18 Error rate comparison of five VAD schemes averaged over five SNRs for $\mathbf{1 2}$ kinds of noises. Error rate: FEC, MSC, OVER, and NDS. Five VAD schemes: AMR1, AMR2, G.729B, LTSV, and LSFM. 
standard deviation of our proposed LSFM-based VAD in terms of MSC, OVER, and NDS are all smaller than that of the LTSV-based VAD.

Thus, in consideration of both accuracy rate and error rate, the proposed VAD algorithm achieved the best compromise when compared with the four representative VADs analyzed.

\section{Conclusions}

The main contribution of this article was the introduction of an efficient long-term spectral flatness measure-based VAD algorithm. The motivation of exploring flatness measure along time frames using a long window was clarified by the LSFM feature distributions as a function of the long-term window length $R$. The discriminative power of the LSFM feature was verified in terms of the separateness of its distribution for noisy speech and non-speech signals. The decision threshold was adapted according to the previous 100 LSFM measures of speech and non-speech. Experiments were done on core TIMIT test set for 12 kinds of noises (11 from NOISEX-92 database and speechshaped noise) across five different SNRs ranging from -10 to $10 \mathrm{~dB}$. No a priori knowledge of noise characteristics was needed for training purposes. The performance of our proposed method was compared with the three standards (namely G.729B, AMR1, and AMR2) and with an emerging LTSV-based VAD algorithm. The results were analyzed by accuracy rate and error rate. Through extensive experiments, we showed that our proposed LSFMbased VAD achieved the best CORRECT, HR0, and NDS and among all tested schemes that averaged all 12 kinds of noises. Furthermore, we investigated the individual performance on each noise type. Our proposed LSFM-based VAD outperformed LTSV-based VAD for 6 out of 12 noise types tested especially for non-stationary impulsive machine gun noise and speechlike babble noise.

The test database used in the implementations was created to simulate typical conversational speech by inserting 2-s silence before and after each utterance from core TIMIT test corpus so that the ratio of speech to nonspeech was almost $40 \%$ to $60 \%$. While this simulates a conversational speech statistically, this is not very realistic in terms of randomness of pauses, hesitations, etc. Furthermore, depending on the choice of the long-term window length ( $R$ and $M$ combination), the LSFM-based VAD application is expected to suffer a delay equal to the duration of the window $(R+M-1$ frames). Therefore, a trade-off between the delay and robustness of VAD should be carefully considered before utilizing the proposed LSFM-based VAD algorithm.

Moreover, it is worth mentioning that there is a tradeoff between HR1 and HR0. The increase of one may lead to a decrease of the other. Therefore, it should be noted that according to different applications, different $(R, M)$ combinations and thresholds for voting scheme can be chosen to meet the variant requirement for HR1 and HR0. For example, HR1 is a crucial factor for speech coding, while high HRO rate is necessary for most speech recognition-oriented systems.

\section{Competing interests}

Both authors declare that they have no competing interests.

Received: 1 February 2013 Accepted: 5 July 2013

Published: 16 July 2013

\section{References}

1. K Itoh, M Mizushima, in IEEE International Conference on Acoustics Speech and Signal Processing (ICASSP), vol. 1. Environmental noise reduction based on speech/non-speech identification for hearing aids (IEEE Piscataway, 1997), pp. 419-422

2. D Freeman, G Cosier, C Southcott, I Boyd, in IEEE International Conference on Acoustics Speech and Signal Processing (ICASSP), vol. 1. The voice activity detector for the Pan-European digital cellular mobile telephone service (IEEE Piscataway, 1989), pp. 369-372

3. F Faubel, M Georges, K Kumatani, A Bruhn, D Klakow, in 2011 Joint Workshop on Hands-free Speech Communication and Microphone Arrays (HSCMA), Edinburgh,30 May-1 June 2011. Improving hands-free speech recognition in a car through audio-visual voice activity detection (IEEE Piscataway, 2011), pp. 70-75

4. W Syed, HC Wu, in Global Telecommunications Conference (GLOBECOM '07), Washington DC, 26-30 November 2007. Speech waveform compression using robust adaptive voice activity detection for nonstationary noise in multimedia communications (IEEE Piscataway, 2007), pp. 3096-3101

5. A Kondoz, B Evans, in 3rd European Conference on Satellite Communications (ECSC-3), Manchester, 2-4 Nov 1993. A high quality voice coder with integrated echo canceller and voice activity detector for VSAT systems (IEEE Piscataway, 1993), pp. 196-200

6. A Benyassine, E Shlomot, HY Su, E Yuen, in IEEE Workshop on Speech Coding For Telecommunications Proceeding. A robust low complexity voice activity detection algorithm for speech communication systems (IEEE Piscataway, 1997), pp. 97-98

7. LR Rabiner, MR Sambur, An algorithm for determining the endpoints of isolated utterances. Bell Syst. Techn. J. 54(2), 297-315 (1975)

8. A Davis, S Nordholm, R Togneri, Statistical voice activity detection using low-variance spectrum estimation and an adaptive threshold. Audio, Speech, Lang. Proc. IEEE Trans. 14(2), 412-424 (2006)

9. Z Shuyin, G Ying, W Buhong, in First International Workshop on Education Technology and Computer Science (ETCS '09), vol. 3. Auto-correlation property of speech and its application in voice activity detection (IEEE Piscataway, 2009), pp. 265-268

10. M Marzinzik, B Kollmeier, Speech pause detection for noise spectrum estimation by tracking power envelope dynamics. Speech and Audio Proc. IEEE Trans. 10(2), 109-118 (2002)

11. E Nemer, R Goubran, S Mahmoud, Robust voice activity detection using higher-order statistics in the LPC residual domain. Speech and Audio Proc. IEEE Trans. 9(3), 217-231 (2001)

12. J Ramirez, J Segura, C Benitez, A Torre, A Rubio, Efficient voice activity detection algorithms using long-term speech information. Speech Commun. 42(3-4), 271-287 (2004)

13. B Lee, M Hasegawa-Johnson, in Proceedings of Biennial on DSP for In-Vehicle and Mobile Systems, Minimum mean squared error a posteriori estimation of high variance vehicular noise. Istanbul, 17-19 June 2007

14. PC Khoa, Noise robust voice activity detection. Master's thesis, NangYang Technological University, 2012

15. P Ghosh, A Tsiartas, S Narayanan, Robust voice activity detection using long-term signal variability. Audio, Speech, and Lang. Proc. IEEE Trans. 19(3), 600-613 (2011)

16. N Madhu, Note on measures for spectral flatness. Electron. Lett. 45(23), 1195-1196 (2009)

17. JS Garofolo, LF Lamel, WM Fisher, JG Fiscus, DS Pallett, NL Dahlgren, V Zue, TIMIT Acoustic-Phonetic Continuous Speech Corpus. (Linguistic Data Consortium, Philadelphia, 1993) 
18. P Renevey, A Drygajlo, in IEEE Proceedings of 7th European Conference on Speech Communication and Technology (EUROSPEECH'2001). Entropy based voice activity detection in very noisy conditions (IEEE Piscataway, 2001), pp. 1887-1890

19. D Manolakis, V Ingle, S Kogon, Statistical and Adaptive Signal Processing: Spectral Estimation, Signal Modeling, Adaptive Filtering, and Array Processing. (Artech House, Norwood, 2005)

20. D Bies, Engineering Noise Control: Theory and Practice. (Taylor \& Francis, New York, 2003)

21. A Varga, HJ Steeneken, Assessment for automatic speech recognition: II. NOISEX-92: a database and an experiment to study the effect of additive noise on speech recognition systems. Speech Commun. 12(3), 247-251 (1993)

22. RV Prasad, A Sangwan, HS Jamadagni, MC Chiranth, R Sah, V Gaurav, in Proceedings of the Seventh International Symposium on Computers and Communications (ISCC'02), Washington, DC. Comparison of voice activity detection algorithms for VolP (IEEE Piscataway, 2002), pp. 530-535

23. F Beritelli, S Casale, G Ruggeri, in Proceedings of Acoustics, Speech, and Signal Processing (ICASSP'01), vol. 3. Performance evaluation and comparison of ITU-T/ETSI voice activity detectors (IEEE Piscataway, 2001), pp. $1425-1428$

24. F Beritelli, S Casale, G Ruggeri, in 5th International Conference on Signal Processing Proceedings (WCCC-ICSP 2000), vol. 2. A psychoacoustic auditory model to evaluate the performance of a voice activity detector (IEEE Piscataway, 2000), pp. 807-810

25. ETSI, Digital Cellular Telecommunications System (Phase 2+); Voice Activity Detector (VAD) for Adaptive Multi-Rate (AMR) Speech Traffic Channels; General Description. (ETSI, Valbonne, 1999)

26. ITU, Coding of Speech at 8 kbit/s Using Conjugate Structure Algebraic CodeExcited Linear Prediction Annex B: A Silence Compression Scheme for G729 Optimized for Terminals Conforming to Recommend V70. (International Telecommunication Union, Geneva, 1996)

27. ETSI, Digital Cellular Telecommunications System (Phase 2+); Adaptive Multi-Rate (AMR) Speech; ANSI-C Code for AMR Speech Codec. (ETSI, Valbonne, 1998)

28. ITU, Coding of Speech at 8 kbit/s Using Conjugate Structure Algebraic CodeExcited Linear Prediction Annex I: Reference Fixed-Point Implementation for Integrating G729 CS-ACELP Speech Coding Main Body with Annexes B, D and E. (International Telecommunication Union, Geneva, 2000)

doi:10.1186/1687-4722-2013-21

Cite this article as: $\mathrm{Ma}$ and Nishihara: Efficient voice activity detection algorithm using long-term spectral flatness measure. EURASIP Journal on Audio, Speech, and Music Processing 2013 2013:21.

\section{Submit your manuscript to a SpringerOpen ${ }^{\circ}$ journal and benefit from:}

- Convenient online submission

- Rigorous peer review

- Immediate publication on acceptance

- Open access: articles freely available online

- High visibility within the field

- Retaining the copyright to your article

Submit your next manuscript at $\boldsymbol{\wedge}$ springeropen.com 